\title{
White matter changes, duration of hypertension, and age are associated with cerebral microbleeds in patients with different stages of hypertension
}

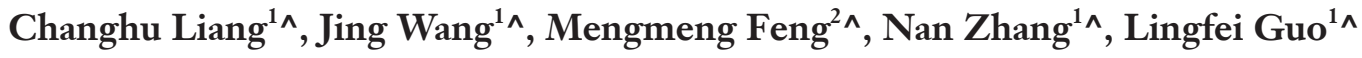 \\ ${ }^{1}$ Department of Radiology, Shandong Provincial Hospital Affiliated to Shandong First Medical University, Jinan, China; ${ }^{2}$ Department of Radiology, \\ Shandong Provincial Hospital, Cheeloo College of Medicine, Shandong University, Jinan, China
}

Contributions: (I) Conception and design: L Guo; (II) Administrative support: C Liang, L Guo; (III) Provision of study materials or patients: L Guo; (IV) Collection and assembly of data: N Zhang, M Feng; (V) Data analysis and interpretation: J Wang; (VI) Manuscript writing: All authors; (VII) Final approval of manuscript: All authors.

Correspondence to: Lingfei Guo. Department of Radiology, Shandong Provincial Hospital Affiliated to Shandong First Medical University, 324 Jingwu Road, Jinan 250021, China. Email: glfsci@163.com.

Background: We aimed to investigate risk factors for the presence and number of cerebral microbleeds $(\mathrm{CMBs})$ in patients with different stages of hypertension stages, with an emphasis on the relationship between white matter changes (WMCs) and CMBs.

Methods: Since 2016, participants aged 40 years or more have been evaluated for the presence of CMBs using enhanced 3D multiecho GE T2*-weighted angiography (ESWAN) sequences. The Mann-Whitney $\mathrm{U}$ test and Pearson $\chi^{2}$ test were used to compare the clinical characteristics between the CMB and no-CMB patient groups. Furthermore, we used Spearman's rank correlation analysis to examine the associations between the degree of CMB severity and other important factors.

Results: CMBs were detected in 110 (36.7\%) of 300 participants. Among patients with stage 2 hypertension, the majority also had CMBs $(61.8 \%, 68 / 110)$. CMBs were positively correlated with age, hypertension stage, duration of hypertension, WMCs, and silent cerebral infarction. Patients with grade 3 WMCs were significantly more likely to have CMBs than those without WMCs; this association was true for both patients with stage 1 and those with stage 2 hypertension. In patients with stage 1 or stage 2 hypertension lasting longer than 20 years, the majority had CMBs (69.0\%, 29/42; 69.1\%, 47/68). The results of binary logistic regression indicated that a more severe hypertension stage, longer duration of hypertension, aging, having silent cerebral infarction and higher values of WMC increase the likelihood of the occurrence of CMBs.

Conclusions: CMBs detected in hypertensive patients were more likely to occur in deep structures, and the grade of WMCs and duration of hypertension were more closely associated with the CMB degree than with age.

Keywords: T2*-weighted; magnetic resonance imaging (MRI); white matter changes (WMCs); cerebral microbleeds (CMBs); hypertension

Submitted Jan 07, 2021. Accepted for publication May 31, 2021.

doi: $10.21037 /$ qims-21-28

View this article at: https://dx.doi.org/10.21037/qims-21-28

$\wedge$ ORCID: Changhu Liang, 0000-0003-3796-2978; Jing Wang, 0000-0003-0346-2000; Mengmeng Feng, 0000-0001-5349-2780; Nan Zhang, 0000-0002-6202-9349; Lingfei Guo, 0000-0002-4885-625X. 


\section{Introduction}

Cerebral microbleeds (CMBs) are defined as focal signal intensity losses that are iron-containing hemosiderin residues of hemoglobin breakdown (1), which can be visualized using $\mathrm{T} 2{ }^{*}$-weighted gradient-recalled-echo (GRE) magnetic resonance imaging (MRI) and are associated with the presence and risk of stroke, regardless of $\mathrm{CMB}$ anatomical distribution or burden $(2,3)$. CMBs are affected by cerebral amyloid angiopathy (CAA) or hypertensive arteriopathy, which may contribute to the development of mixed CMBs (4).

White matter changes (WMCs) are defined as areas with high signal intensity that are visible using T2-weighted or fluid-attenuated inversion recovery (FLAIR) MRI and as areas of low attenuation on CT images. The mechanisms by which WMCs develop are not fully understood and may include degeneration of myelin and axons with increased intracellular and extracellular water content, gliosis and infarction.

Age, diagnosis with intracerebral hemorrhage (ICH) or ischemic stroke, dementia, hypertension, and use of antithrombotic therapy have been associated with a higher risk of $\mathrm{CMB}$ detection using traditional 2D T2*-weighted GRE imaging patients $(5,6)$. Both CMBs and WMCs are the main imaging markers of cerebral small vessel disease (7). They are closely related and have the same pathological characteristics and often occur in patients with hypertension (8-10). Although CMBs and WMCs may occur with increased frequency in hypertension $(11,12)$, knowledge of their relationship with the duration of hypertension in patients in different stages of hypertension is limited. Some studies have reported WMCs in subjects displaying CMBs in patients with hypertension (13), but no study has primarily focused on the WMC-CMB relationship in patients with different stages of hypertension.

We performed an enhanced 3D multiecho GE T2*weighted angiography (ESWAN) sequence to detect CMBs and investigated the risk factors associated with the presence and number of CMBs among patients with different stages of hypertension, emphasizing the relationship between WMCs and CMBs.

\section{Methods}

\section{Participants}

The study was conducted in accordance with the Declaration of Helsinki (as revised in 2013). This study was approved by the Institutional Review Board of Shandong Medical Imaging Research Institute Affiliated with Shandong University, and all methods were carried out in accordance with relevant guidelines and regulations. All participants were informed of the detailed experimental procedures and signed informed consent forms. Between July 2016 and May 2018, all consecutive patients who were evaluated for hypertension were screened for participation in the present study. The inclusion criteria for this study were hypertensive patients over 40 years old. Patients must have undergone MRI examinations based on clinical symptoms suggestive of central nervous system damage (such as headache, migraine, tinnitus, insomnia, limb numbness, or transient ischemic attack symptoms), cognitive function disorders, lacunar infarction, health-screening brain tests performed at our institute. Patients meeting these criteria were considered potential subjects in this study. Patients were diagnosed according to the hypertension diagnosis standard (The Seventh Report of the Joint National Committee on Prevention, Detection, Evaluation, and Treatment of High Blood Pressure, The JNC 7 Report, Table 1) (14). We divided the hypertensive patients into two groups according to their hypertension stage (1 or 2 ) and divided them into a group with hypertension $\leq 20$ years and a group with hypertension $>20$ years. Patients were excluded from the study if conventional MRI revealed acute ICH, dementia, consciousness disorders, cerebral vasculitis, cerebral tumor, cerebral trauma, cavernous hemangioma, or arteriovenous malformation.

In total, 327 participants were eligible for our study; of those, 320 participants agreed to participate and provided written informed consent. In 11 participants, imaging could not be completed due to physical problems; in 9 cases, motion artifacts prevented proper image assessment. In total, 300 hypertensive participants (160 males and 140 females) were included in the analysis. The average patient age was $61.70 \pm 10.31$ years (range: $40-85$ years). The average amount of time that passed since the patients had been diagnosed with hypertension was $17.00 \pm 8.10$ years (range: $1-43$ years).

\section{MR protocols}

Imaging was performed using a 3.0-T MRI scanner (General Electric Medical Systems, USA) with an eight-channel head coil. Two technologists performed all the MR imaging examinations according to a standardized conventional protocol. The parameters for the MR sequences with spin 
Table 1 Blood pressure classification for adults (JNC 7 Express)

\begin{tabular}{lcc}
\hline Blood pressure classification & SBP $(\mathrm{mmHg})$ & DBP $(\mathrm{mmHg})$ \\
\hline Normal & $<120$ & And $<80$ \\
Prehypertension & $120-139$ & Or 80-89 \\
Stage 1 hypertension & $140-159$ & Or 90-99 \\
Stage 2 hypertension & $>160$ & Or $>100$ \\
\hline
\end{tabular}

SBP, systolic blood pressure; DBP, diastolic blood pressure; JNC 7 Express, the Seventh Report of the Joint National Committee on Prevention, Detection, Evaluation, and Treatment of High Blood Pressure, U.S. National Institutes of Health-National Heart, Lung, and Blood Institute.

echo FLAIR T1-weighted images were as follows: repetition time (TR), $1977 \mathrm{~ms}$; echo time (TE), $27.7 \mathrm{~ms}$; and inversion time (TI), $860 \mathrm{~ms}$. The following parameters were used for fast spin echo T2-weighted images: TR, 3,280 ms and TE, $106.9 \mathrm{~ms}$ and for T2 FLAIR: TR, 9,002 ms; TE, $155.7 \mathrm{~ms}$; and TI, 2,250 ms. The parameters for ESWAN were as follows: TR, $43.8 \mathrm{~ms}$; TE, $5.6 \mathrm{~ms}$; flip angle, $20^{\circ}$; bandwidth, $62.5 \mathrm{~Hz}$; slice thickness, $2 \mathrm{~mm}$; slice gap, $0 \mathrm{~mm}$; matrix, $480 \times 388$; field of view, $240 \times 240 \mathrm{~mm}^{2}$; and time of examination: approximately 6 minutes.

The images were processed using ESWAN software in Functool according to the manufacturer's instructions. All images were transferred to an ADW4.5 workstation for ESWAN postprocessing. As part of the reconstruction processes, varying phase shifts arising predominantly from background field heterogeneity and air-tissue interfaces were removed.

\section{Evaluation of blood pressure}

Conventional office blood pressure (COBP) was measured by a nurse with a conventional, calibrated, mercury sphygmomanometer from the sitting individual's right arm after a 10-minute rest. The last 5 minutes of rest were spent in the measuring room with the cuff around the right upper arm. BP was measured using a pressure cuff of appropriate size and methods. Systolic BP and diastolic BP were defined according to Korotkoff sounds I and V. Means of 2 measurements performed at a 2-minute interval were used to determine office $\mathrm{BP}(15-17)$.

\section{Rating of CMBs and WMCs}

In this study, CMBs were defined as homogeneous round foci, $\leq 10 \mathrm{~mm}$ in diameter (no minimum size was specified), of low signal intensity on GRE T2*-weighted (or ESWAN)
MRI and excluding symmetrical low-signal intensity in the globus pallidus or empty flow in the distal-side branch cross at the cerebral artery branch $(18,19)$. Vascular flow voids and basal ganglia mineralization were also excluded. The following seven regions were observed: the cortex, subcortical white matter, external capsule (EC)/internal capsule (IC), thalamus, basal ganglia gray matter, brainstem, and cerebellum. The cortex included subcortical regions touching the gray-white matter junction; the subcortical regions included the periventricular white matter and deep portions of the centrum semiovale; and basal ganglia gray matter included the caudate and lentiform nuclei. CMBs were classified by degree and assigned scores based on the total number of lesions as follows: $0=$ absent, $1=$ mild $(1$ to 2), $2=$ moderate $(3$ through 10$)$, and $3=$ severe $(>10)(20)$.

According to the Standards for Reporting Vascular Changes on Neuroimaging (STRIVE) criteria (21), WMCs were signal abnormalities of variable size, defined as white matter hyperintense lesions on FLAIR images. Lacunes were round or ovoid, subcortical small lesions $(3-15 \mathrm{~mm}$ in diameter) that were hypointense on $\mathrm{T} 1 \mathrm{~W}$ images and hyperintense on $\mathrm{T} 2 \mathrm{~W}$ images and had a perilesional halo on FLAIR images. The severity of lacunes and WMCs was assessed using a simple scoring method. The following rating scores were used to classify WMCs (0-3): Grade $0=$ no lesions (including symmetrical, well-defined caps or bands); Grade 1 = focal lesions; Grade 2 = beginning confluence of lesions; and Grade 3 = diffuse involvement of the entire region, with or without the involvement of $U$ fibers $(19,22)$.

The acquisition date and participant identification were removed from all images. ESWAN images were randomly allocated to one of four reviewers who were blinded to the other sequence and to all clinical information. Reviewers independently rated the presence, location, and number of all CMBs. All studies with a potential microbleed 
were reviewed for confirmation by an experienced neuroradiologist.

\section{Statistical analysis}

All statistical analyses were performed using SPSS 17.0 software. To compare the clinical characteristics between the $\mathrm{CMB}$ and no-CMB groups, the Mann-Whitney $\mathrm{U}$ test and Pearson's $\chi^{2}$ test were used. To compare the differences in the WMC grade, duration of hypertension, and age between the CMB and no-CMB groups at different stages of hypertension, Pearson's $\chi^{2}$ test was used as appropriate. To detect the difference in the distribution of CMBs between hypertension stage 1 and 2 patients, we applied Pearson's $\chi^{2}$ statistic or Fisher's exact test for categorical variables. Furthermore, using Spearman's rank correlation analyses, we examined the association between the degree of CMBs and WMC grade, duration of hypertension, and age of patients at different stages of hypertension. A probability (P) value $<0.05$ was considered significant. Conditional forward method binary logistic regression analysis was used to establish a mathematical model of the relationship between the variables and CMBs. The significance level was set at $\alpha=0.05$.

\section{Results}

The details of the demographic and MRI findings of the participants are listed in Table 2. CMBs were detected in $110(36.7 \%)$ of the 300 participants and in $75(68.2 \%)$ of participants over 60 years old. CMBs were similarly prevalent among men and women, with $59(53.6 \%)$ of 160 cases in men and $51(46.4 \%)$ of 140 cases in women $(\mathrm{P}=0.09)$.

In patients with hypertension stage 1 , the rate of cases with $\mathrm{CMBs}$ was significantly lower than the percentage of cases without $\mathrm{CMBs}(\mathrm{P}=0.002)$; in contrast, the rate of CMBs was significantly higher than that of cases without CMBs in patients with stage 2 hypertension $(\mathrm{P}=0.004)$. There were significant differences in the average duration of hypertension between the participants with CMBs (25 years) and those without CMBs (16 years). In the patients diagnosed with silent cerebral infarction $(n=70)$, the percentage of patients with $\mathrm{CMBs}$ were significantly higher than that of patients without $\mathrm{CMBs}(\mathrm{P}=0.001)$.

Furthermore, we compared the differences between the severity of WMCs, duration of hypertension, age, and presence of CMBs in patients with different stages of hypertension (Table 3). In patients with grade $3 \mathrm{WMCs}$, the percentage of patients with CMBs was significantly higher than that in patients without CMBs $(\mathrm{P}<0.05)$ in patients with both stage 1 and stage 2 hypertension. In the >20 years group, the percentage of patients with CMBs was significantly higher than that of patients without $\mathrm{CMBs}$ for both stage 1 and stage 2 hypertensive patients $(\mathrm{P}<0.05)$. When comparing middle-aged and elderly patients, there were no significant differences in the percentage of patients with $\mathrm{CMBs}$ and those without $\mathrm{CMBs}(\mathrm{P}>0.05)$.

We examined the association between the degree $(0-3)$ of CMBs and the grade of WMCs (0-3), duration of hypertension (1-10, 11-20, 21-30, 31-40, >40 years), and age (40-50, 51-60, 61-70, 71-80, >80 years) among patients with different stages of hypertension using Spearman's rank correlation analyses (Table 4). In patients with stage 2 hypertension, we found that the correlation between the duration of hypertension and both the degree of CMBs and grade of WMCs was highly positive ( $\mathrm{r}=0.712, \mathrm{P}=0.003$ and $\mathrm{r}=0.664, \mathrm{P}=0.034$, respectively) (Figures 1,2 ). In patients with stage 1 hypertension, we found no correlation between these characteristics. In all participants, the correlation between the degree of CMBs and age was moderately positive in those with stage 1 or 2 hypertension.

According to the difference and correlation analysis results (Tables 3,4) and combined with the appropriate application conditions for binary logistic regression, age, hypertension stage, total cholesterol, creatinine, uric acid, WMC, silent cerebral infarction and duration of hypertension were introduced to the binary logistic regression analysis (Table 5). Finally, the results indicated that a more severe hypertension stage, longer duration of hypertension, aging, silent cerebral infarction and higher values of WMC increased the likelihood of the occurrence of CMBs.

In the participants with CMBs and hypertension stage 1 or 2, CMBs were most commonly found in the basal ganglia gray matter and subcortical white matter. Additionally, the number of CMBs in the basal ganglia gray matter and brainstem was higher in patients with stage 2 hypertension than in those with stage $1(\mathrm{P}<0.05)$ (Table 6).

\section{Discussion}

CMBs represent focal accumulations of hemosiderincontaining macrophages with paramagnetic properties causing signal loss because of susceptibility effects (23). Histopathologic studies have shown that CMBs are 
Table 2 Clinical characteristics of the study participants according to CMBs status

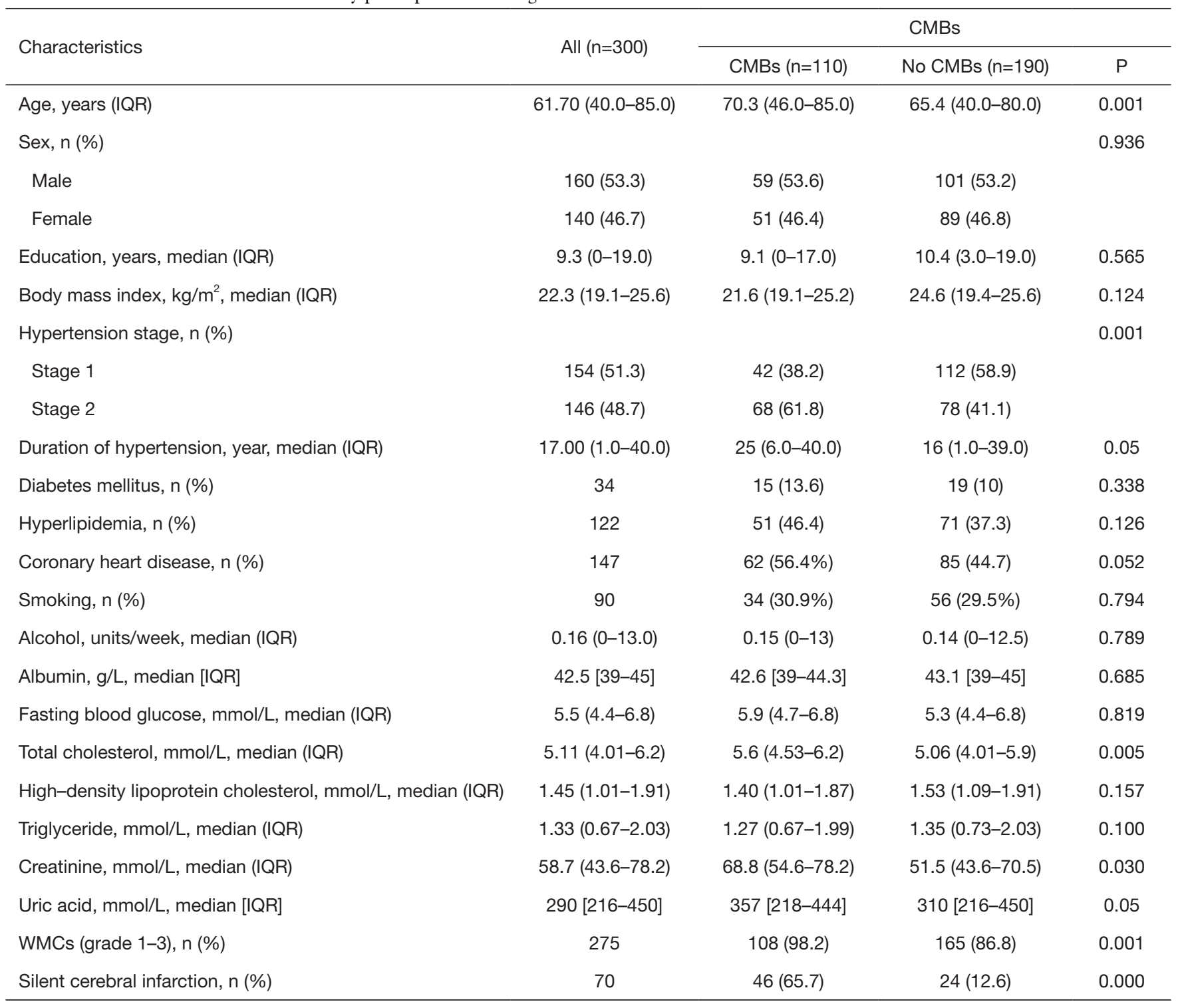

CMBs, cerebral microbleeds; WMCs, white matter changes.

affected by moderate to severe fibrohyalinosis and arteriolosclerosis (24). Although the predictive value of CMBs for stroke prognosis has not been widely accepted, several clinical studies have suggested that CMBs might be associated with an increased risk of recurrent stroke $(2,25,26)$. One approach to interpreting CMBs is to regard them as a marker of accompanying vascular pathological changes. Histopathological analyses of the vessels associated with CMBs have generally identified two types of vascular pathological changes, hypertensive vasculopathy and CAA, which are distinguishable according to the location of the CMBs. In the past year, a new scan study suggested that $\mathrm{CMB}$ s visible using $\mathrm{T} 2{ }^{*}$ weighted MRI were a possible marker of both CAA and hypertensive vasculopathy progression (27). CMBs are often accompanied by elevated markers of vascular inflammation dysfunction and systemic inflammation, particularly in stroke patients (9).

Hypertension is a major risk factor associated with bloodbrain barrier leakage of plasma components and may be a cause of WMCs and lacunar stroke (28). Recent studies have suggested that CMBs are frequently found in hypertensive 
Table 3 Difference in the severity of WMC, duration, age and the presence of CMBs among patients with different stages of hypertension

\begin{tabular}{|c|c|c|c|c|c|c|}
\hline Hypertension stage & \multicolumn{2}{|c|}{ WMCs grade } & \multicolumn{2}{|c|}{ Duration of hypertension (years) } & \multicolumn{2}{|c|}{ Age (years) } \\
\hline \multicolumn{7}{|l|}{ Stage $1(n=154)$} \\
\hline CMBs $(n=42)$ & $17(40.5 \%)$ & $25(59.5 \%)$ & $13(31.0 \%)$ & $29(69.0 \%)$ & $20(47.6 \%)$ & $22(52.4 \%)$ \\
\hline No-CMBs $(n=112)$ & $64(57.1 \%)$ & $48(42.9 \%)$ & $76(67.9 \%)$ & $36(32.1 \%)$ & $54(48.2)$ & $58(51.8 \%)$ \\
\hline \multicolumn{7}{|l|}{ Stage $2(n=146)$} \\
\hline CMBs $(n=68)$ & $22(32.4 \%)$ & $46(67.6 \%)$ & $21(30.9 \%)$ & $47(69.1 \%)$ & $19(27.9 \%)$ & $49(72.1 \%)$ \\
\hline No-CMBs $(n=78)$ & $40(51.3 \%)$ & $38(48.7 \%)$ & $45(57.7 \%)$ & $33(42.3 \%)$ & $30(38.5 \%)$ & $48(61.5 \%)$ \\
\hline Statistical values & \multicolumn{2}{|c|}{$\chi^{2}=5.328, P=0.021$} & \multicolumn{2}{|c|}{$\chi^{2}=10.542, P=0.001$} & \multicolumn{2}{|c|}{$\chi^{2}=1.803, P=0.179$} \\
\hline
\end{tabular}

CMBs, cerebral microbleeds; WMCs, white matter changes.

Table 4 Correlation of the degree of CMBs and grade of WMCs, duration of hypertension, and age among patients with different stages of hypertension

\begin{tabular}{lccc}
\hline Hypertension stage & WMCs grade $(0-3)$ & Duration of hypertension (1-40 years) & Age $(40-85$ years $)$ \\
\hline Stage 1 $(n=42)$ & 0.396 & 0.297 & 0.423 \\
$r$ & 0.09 & 0.154 & 0.050 \\
$P$ & & & 0.435 \\
Stage 2 $(n=68)$ & 0.712 & 0.664 & 0.05 \\
$r$ & 0.003 & 0.034 & \\
$P$
\end{tabular}

CMBs, cerebral microbleeds; WMCs, white matter changes.
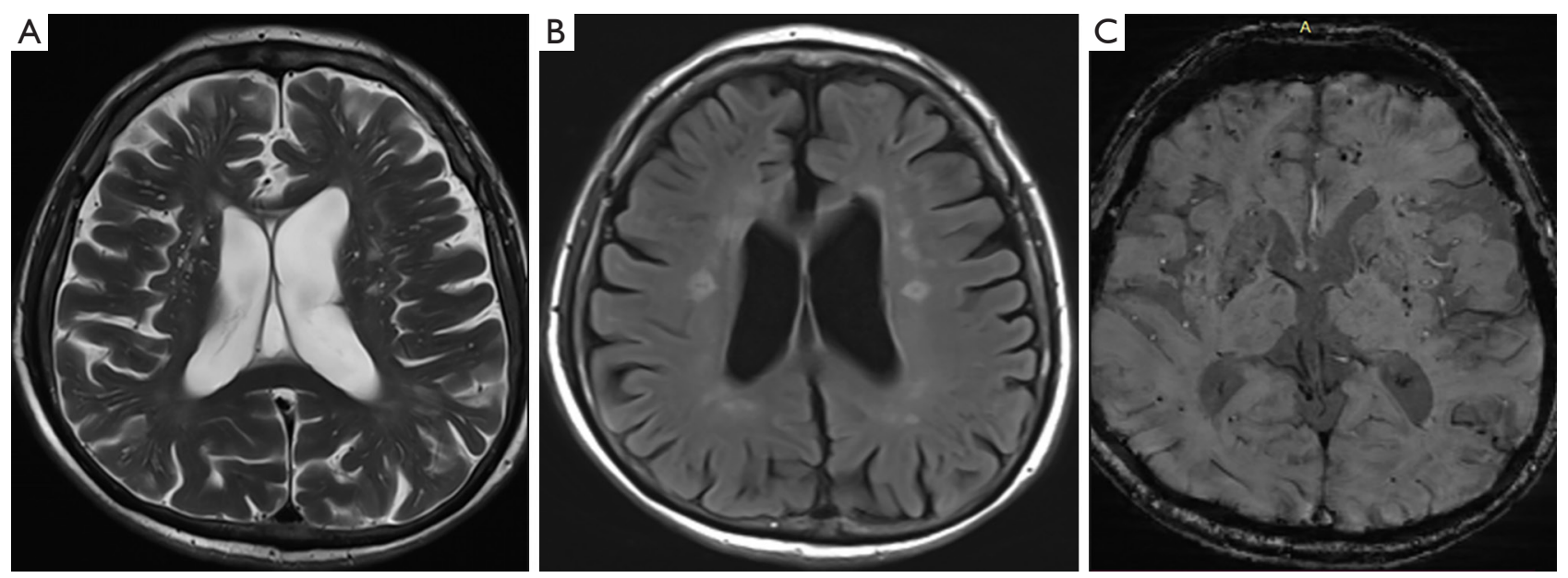

Figure 1 A 65 -year-old male with stage 2 hypertension. Axial T2WI and T2 FLAIR MR image showing white matter lesions in the entire region around the lateral ventricle (A,B). The patient was scored as WMCs grade 3. An axial ESWAN MR image showing multiple CMBs in both cerebral hemispheres. The CMB degree was "severe" (C). CMBs, cerebral microbleeds; WMCs, white matter changes. ESWAN, enhanced 3D multiecho GE T2*-weighted angiography. 

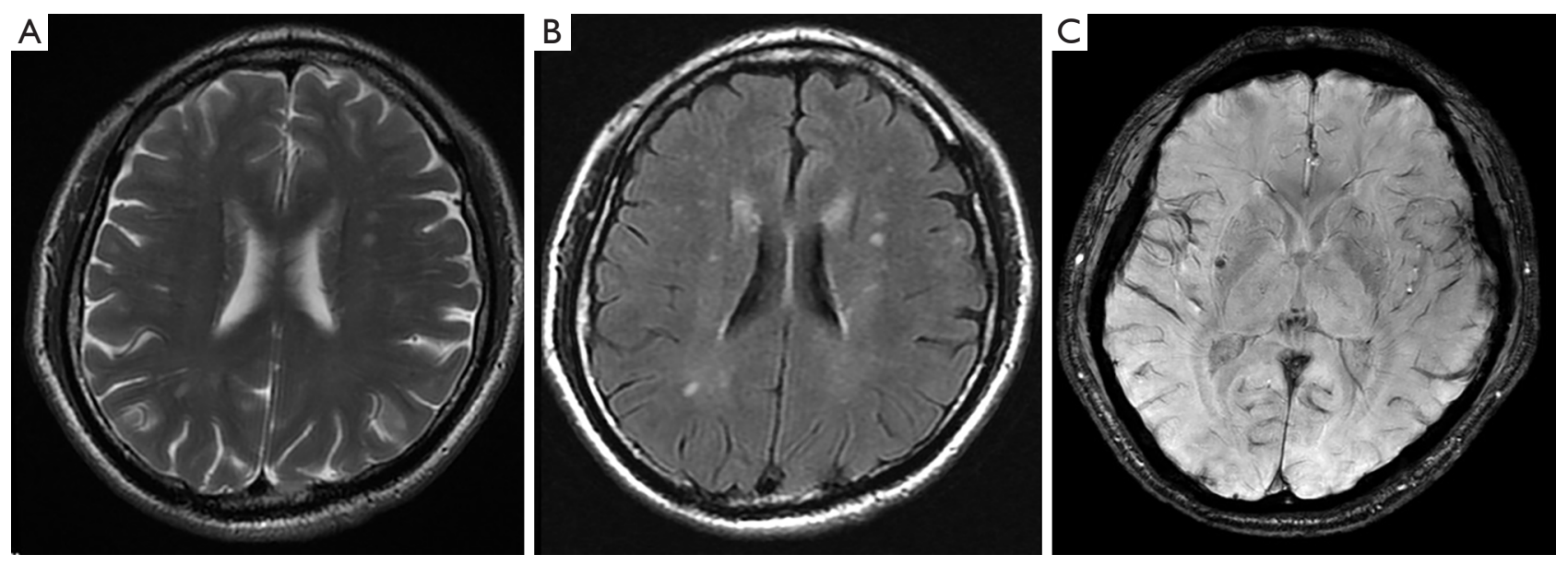

Figure 2 A 58-year-old female with stage 2 hypertension. Axial T2WI and T2 FLAIR MR image showing the beginning confluence of lesions around the lateral ventricle. The patient was scored as WMCs grade 2 (A,B). An axial ESWAN MR image showing a single CMB in the right basal ganglia. The CMBs degree was "mild" (C). CMBs, cerebral microbleeds; WMCs, white matter changes. ESWAN, enhanced 3D multiecho GE T2*-weighted angiography.

Table 5 Binary logistic regression results of the risk factors related to the occurrence of CMBs

\begin{tabular}{|c|c|c|c|c|c|c|}
\hline Variables & $\mathrm{B}$ & SE & $\chi^{2}$ & OR & $95 \% \mathrm{Cl}$ & $P$ \\
\hline Age & 0.952 & 0.315 & 7.954 & 2.592 & $1.398-4.808$ & $<0.001$ \\
\hline Hypertension duration & 0.969 & 0.336 & 4.431 & 2.634 & $1.069-6.942$ & 0.002 \\
\hline Silent cerebral infarction & 0.854 & 0.408 & 4.382 & 2.349 & $1.056-5.225$ & 0.030 \\
\hline
\end{tabular}

Hypertension stage (stage $1=0$, stage $2=1$ ), WMCs (grade $0-2=0$, grade $3=1$ ), silent cerebral infarction (yes $=1$, no =0) and duration of hypertension ( $1-20$ years $=0,>20$ years $=1$ ). CMBs, cerebral microbleeds; WMCs, white matter changes; $B$, coefficient value; SE, standard error; $\mathrm{Cl}$, confidence interval; $\mathrm{OR}$, odds ratio.

Table 6 Difference in CMBs distribution according to brain location in patients with different stages of hypertension

\begin{tabular}{lccccccc}
\hline & \multicolumn{5}{c}{ Location } & & \\
\cline { 2 - 7 } Hypertension stage & Cortex & $\begin{array}{c}\text { Subcortical } \\
\text { white matter }\end{array}$ & $\begin{array}{c}\text { External capsule/ } \\
\text { internal capsule }\end{array}$ & $\begin{array}{c}\text { Basal ganglia } \\
\text { gray matter }\end{array}$ & Thalamus & Brainstem & Cerebellum \\
\hline Stage 1 $(\mathrm{n}=154)$ & $28(18.2 \%)$ & $77(50.0 \%)$ & $49(31.8 \%)$ & $76(49.3 \%)$ & $31(20.1 \%)$ & $10(6.5 \%)$ & $11(7.1 \%)$ \\
Stage 2 $(\mathrm{n}=146)$ & $30(20.5 \%)$ & $76(52.1 \%)$ & $51(34.9 \%)$ & $90(61.6 \%)$ & $33(22.6 \%)$ & $17(11.6 \%)$ & $10(6.8 \%)$ \\
$P$ & $>0.05$ & $>0.05$ & $>0.05$ & $<0.05$ & $>0.05$ & $<0.05$ & $>0.05$ \\
\hline
\end{tabular}

Percentages do not total $100 \%$ because some patients had CMBs in more than one location. CMBs, cerebral microbleeds. 
patients without a history of cerebrovascular disease and are independently associated with higher daytime and nighttime BP levels (29). The present study demonstrated that in this population, the prevalence of CMBs was $36.7 \%$. The prevalence of CMBs in our hypertensive cohort was approximately six times higher than that reported in the general population and was approximately two times higher than that in patients without a history of cerebrovascular disease (30); these results are consistent with the incidence of CMBs in Chinese hypertensive patients. Importantly, the CMB count was not influenced by a history of cerebral tumor, cerebral trauma, encephalic angioma, or arteriovenous malformation because patients with these diseases were excluded from our study.

The prevalence of $\mathrm{CMBs}$ has been investigated in various populations. In elderly subjects lacking cerebrovascular diseases, the $\mathrm{CMB}$ prevalence is between $5 \%$ and $6 \%(31)$; Tsushima et al. reported that the CMB incidence was 3.1\% in 450 neurologically healthy Japanese adults. In addition, one study reported that $\mathrm{CMB}$ prevalence was closely related to hypertension and heavy cigarette smoking (32). The frequency of CMBs in patients with stroke and healthy older subjects reported in our study is consistent with previously reported results (33). The very high frequencies of stroke reported in some previous studies (e.g., 78\%) may reflect patient selection bias, with experiments only including subjects with hemorrhagic stroke. The prevalence of CMBs in our study population seems to be higher than these previous studies, which is attributable to hypertension in our subjects and/or higher accuracy in detecting CMBs through the use of a high-field system (3T MRI) $(34,35)$.

In our participants, we found an association between $\mathrm{CMBs}$ and age, hypertension stage, duration of hypertension, silent cerebral infarction, WMCs, coronary heart disease, total cholesterol, creatinine, and uric acid. The strengths of the present study include analyses of potential confounders such as age, duration of hypertension, and WMCs. Earlier studies have highlighted the relationship between CMBs and some conventional factors (e.g., sex, age, BP, smoking, white matter lesions, and lacunar infarction visible using MRI) $(33,36)$; however, these studies only evaluated factors for ischemic or hemorrhagic diseases. To address this issue, we divided our participants into 2 groups based on hypertension stage, allowing us to evaluate whether there was a significant correlation between the incidence of CMBs and conventional risk factors including age, WMCs, and duration of hypertension.

The distributions of low-grade and high-grade
WMCs differed between patients with different stages of hypertension. In patients with stage 1 hypertension, the rate of grade 3 or grade $0-2$ CMBs was no higher than the rate without CMBs. In contrast, the rate of CMBs was higher in patients with higher hypertension stages. When comparing the duration of hypertension between patients with CMBs and those without CMBs, the same result was found. These results suggest that the risk of CMBs is much higher in patients with higher BP levels, regardless of the duration of hypertension or degree of WMCs. Henskens et al. performed a similar study and concluded that CMBs were frequently found in hypertensive patients without a history of cerebrovascular disease and were independently associated with higher daytime and nighttime BP levels (29). The rate of CMBs in patients with stage 1 hypertension was not different between those who were middle-aged and older; the same held true for patients with stage 2 hypertension.

In our study, to facilitate research classification, we divided the patients into two groups according to JNC 7, which suggests that all people with hypertension (stages 1 and 2) should be treated. We chose to emphasize the relationship between age, duration of hypertension, and degree of WMCs and CMBs because these risk factors are easy to determine through interviews and the evaluation of MR or CT images taken during diagnosis or treatment. By preliminarily assessing the risk of CMBs in hypertensive patients on the basis of these factors, the radiologist targeted some patients for $\mathrm{T} 2{ }^{*}$-weighted sequence MRI, and the neurologist was able to consider the existence of CMBs in patients with stage 2 hypertension during treatment.

In patients with stage 1 hypertension, a higher degree of CMBs was associated with some risk factors, including WMC grade, duration of hypertension, and age, but the correlations between these factors and CMBs were not strong. The results suggest that there were fewer CMBs in patients with lower BP, which may indirectly explain how these factors influence small arteries. In patients with stage 2 hypertension, the correlations between the risk factors and the degree of CMBs were stronger than those in patients with stage 1 . These factors may have a larger effect on the presence of CMBs, but the relationship between age and $\mathrm{CMBs}$ did not change much relative to that in patients with stage 1 hypertension.

Additionally, we confirmed the close association among the number of CMBs, the severity of WMCs, and the duration of hypertension. Our results suggest that the risk associated with age was confounded by the risk associated 
with WMCs and that age had a weaker correlation with CMBs than both WMCs and the duration of hypertension. Age has been reported as one of the most important risk factors for not only CMBs but also high-grade WMCs $(37,38)$. In these studies, advanced WMCs were assessed under similar conditions, consisting of extensive white matter damage from cerebral small vessel diseases such as microangiopathy and amyloid angiopathy.

The possibility that all diffuse CMBs are the result of hypertensive microangiopathies must be considered. Indeed, chronic hypertension can influence the development of hypertensive microangiopathies anywhere in the brain (39), but CAA is typically found in cortical or leptomeningeal regions and is not necessarily related to hypertension (40). Histopathologic analyses of CMBs that are visible using MRI have shown that CMBs reflect widespread involvement of the arterioles and are caused by hypertension, amyloid deposition, or both pathogenetic mechanisms. Thus, both pathogeneses might be involved in diffuse CMBs. CAA and mixed CMBs show different clinical and radiological profiles in the absence of macrohemorrhages (41).

We found that CMBs occurring in deep structures were more common than those found in the lobar hemispheres of our participants. There were significantly more CMBs in the basal ganglia of hypertension stage 2 patients than in stage 1 patients. A similar result was true for the brainstem. These results suggest that CMBs in the brainstem should be considered when treating patients with stage 2 hypertension. Previous research showed that CMBs occurred more frequently in deep structures than in lobes (42), which is consistent with our study. The lobar hemispheres are common sites for hypertensive cerebral hemorrhages. Accordingly, these results suggest that modifiable risk factors such as high $\mathrm{BP}$ can be associated with CMBs.

The present study has several limitations, including its cross-sectional design and the relatively small number of participants with CMBs. Although the group size was sufficient to perform the analyses, additional exploratory adjustments need to be interpreted within the context of their statistical limitations. In this study, we did not list hypertensive patients who met the diagnostic criteria of CAA separately. The "gold standard" of CAA is to make a pathological diagnosis through autopsy, which is another limitation of our study. We will focus on patients clinically diagnosed with CAA in future research. Our findings require confirmation in longitudinal and adequately powered studies. Another limitation is the lack of a local, preferably community-based, control population. Such a control group would have enabled us to assess whether the prevalence of CMBs in our hypertensive cohort was higher than that in previous studies.

Our study showed that CMBs frequently occur in patients. Moreover, the data suggest that participants with stage 2 hypertension are more likely to display CMBs on MRI than participants with stage 1 . In contrast to the general belief that $\mathrm{CMBs}$ are clinically silent, several large clinical studies have established an association of CMBs with vascular and systemic inflammation (43) as well as with cognitive decline in patients with vascular dementia and Alzheimer's disease (AD) (44-46) and an increased risk of stroke recurrence, which illustrates the potential clinical relevance of these small lesions (47). We postulate that CMBs should be considered an independent marker of hypertensive target organ damage in the brain. Our findings require confirmation in adequately powered and preferably long-term follow-up studies, which should also address the role of CMBs in risk estimation and the prevention of both future strokes and impaired brain function.

\section{Conclusions}

In conclusion, the CMBs observed in hypertensive patients occurred more frequently in deep structures, and both the WMC grade and duration of hypertension had a closer association with the degree of CMBs than age. These results should be fully considered during clinical treatment. CMBs in hypertensive patients were more common in deep structures, and both the WMC grade and duration of hypertension were more closely associated with the degree of CMBs than with age.

\section{Acknowledgments}

Funding: This work was supported by grants from the Natural Science Foundation of Shandong Province (ZR2020MH288), Technology Development Plan of Jinan (201302049, 201602206, 201907052), Medical and Health Science and Technology Development Project of Shandong Province (2016WS0529, 2019WS544) and Funding for Study Abroad Program by Shandong Province (201803059, 201803010).

\section{Footnote}

Conflicts of Interest: All authors reviewed the manuscript. 
All authors have completed the ICMJE uniform disclosure form (available at https://dx.doi.org/10.21037/qims-21-28). The authors have no conflicts of interest to declare.

Ethical Statement: The authors are accountable for all aspects of the work in ensuring that questions related to the accuracy or integrity of any part of the work are appropriately investigated and resolved. The study was conducted in accordance with the Declaration of Helsinki (as revised in 2013). This study was approved by the Institutional Review Board of Shandong Medical Imaging Research Institute Affiliated with Shandong University, and all methods were carried out in accordance with relevant guidelines and regulations. All participants were informed of the detailed experimental procedures and signed informed consent forms.

Open Access Statement: This is an Open Access article distributed in accordance with the Creative Commons Attribution-NonCommercial-NoDerivs 4.0 International License (CC BY-NC-ND 4.0), which permits the noncommercial replication and distribution of the article with the strict proviso that no changes or edits are made and the original work is properly cited (including links to both the formal publication through the relevant DOI and the license). See: https://creativecommons.org/licenses/by-nc-nd/4.0/.

\section{References}

1. Schrag M, McAuley G, Pomakian J, Jiffry A, Tung S, Mueller C, Vinters HV, Haacke EM, Holshouser B, Kido D, Kirsch WM. Correlation of hypointensities in susceptibilityweighted images to tissue histology in dementia patients with cerebral amyloid angiopathy: a postmortem MRI study. Acta Neuropathol 2010;119:291-302.

2. Wilson D, Ambler G, Lee KJ, Lim JS, Shiozawa M, Koga M, et al. Cerebral microbleeds and stroke risk after ischaemic stroke or transient ischaemic attack: a pooled analysis of individual patient data from cohort studies. Lancet Neurol 2019;18:653-65.

3. Shuaib A, Akhtar N, Kamran S, Camicioli R. Management of Cerebral Microbleeds in Clinical Practice. Transl Stroke Res 2019;10:449-57.

4. Ii Y, Ishikawa H, Matsuyama H, Shindo A, Matsuura K, Yoshimaru K, Satoh M, Taniguchi A, Matsuda K, Umino M, Maeda M, Tomimoto H. Hypertensive Arteriopathy and Cerebral Amyloid Angiopathy in Patients with Cognitive Decline and Mixed Cerebral Microbleeds. J
Alzheimers Dis 2020;78:1765-74.

5. Loitfelder M, Seiler S, Schwingenschuh P, Schmidt R. Cerebral microbleeds: a review. Panminerva Med 2012;54:149-60.

6. Wilson D, Werring DJ. Antithrombotic therapy in patients with cerebral microbleeds. Curr Opin Neurol 2017;30:38-47.

7. Gao Z, Wang W, Wang Z, Zhao X, Shang Y, Guo Y, Gong M, Yang L, Shi X, Xu X, An N, Wu W. Cerebral microbleeds are associated with deep white matter hyperintensities, but only in hypertensive patients. PLoS One 2014;9:e91637.

8. Charidimou A, Boulouis G, Haley K, Auriel E, van Etten ES, Fotiadis P, Reijmer Y, Ayres A, Vashkevich A, Dipucchio ZY, Schwab KM, Martinez-Ramirez S, Rosand J, Viswanathan A, Greenberg SM, Gurol ME. White matter hyperintensity patterns in cerebral amyloid angiopathy and hypertensive arteriopathy. Neurology 2016;86:505-11.

9. Low A, Mak E, Rowe JB, Markus HS, O'Brien JT. Inflammation and cerebral small vessel disease: A systematic review. Ageing Res Rev 2019;53:100916.

10. Wang PN, Chou KH, Peng LN, Liu LK, Lee WJ, Chen LK, Lin CP, Chung CP. Strictly Lobar Cerebral Microbleeds Are Associated with Increased White Matter Volume. Transl Stroke Res 2020;11:29-38.

11. Ni R, Chu L, Xu D, Li Y, Li Y, Zhang Y, You M, Zhu Y, Ouyang F, Zhang J, Cai G, Lin X, Yang Z, Gao Z. Risk factors of cerebral microbleeds in young and middle-aged patients with hypertension. Neurol Res 2018;40:413-8.

12. Elmståhl S, Ellström K, Siennicki-Lantz A, Abul-

Kasim K. Association between cerebral microbleeds and hypertension in the Swedish general population "Good Aging in Skåne" study. J Clin Hypertens (Greenwich) 2019;21:1099-107.

13. Li S, Fang F, Cui M, Jiang Y, Wang Y, Kong X, Tian W, Fan M, Yuan Z, Chen J, Yang Q, Xue F, Wang J, Lu M, Wang $\mathrm{X}$, Chen $\mathrm{X}$, Jin L, Ye W. Incidental findings on brain MRI among Chinese at the age of 55-65 years: the Taizhou Imaging Study. Sci Rep 2019;9:464.

14. Lenfant C, Chobanian AV, Jones DW, Roccella EJ. Seventh report of the Joint National Committee on the Prevention, Detection, Evaluation, and Treatment of High Blood Pressure (JNC 7): resetting the hypertension sails. Hypertension 2003;41:1178-9.

15. Parati G, Stergiou GS, Asmar R, Bilo G, de Leeuw P, Imai $Y$, et al. European Society of Hypertension practice guidelines for home blood pressure monitoring. J Hum 
Hypertens 2010;24:779-85.

16. Niiranen TJ, Hänninen MR, Johansson J, Reunanen A, Jula AM. Home-measured blood pressure is a stronger predictor of cardiovascular risk than office blood pressure: the Finn-Home study. Hypertension 2010;55:1346-51.

17. Andreadis EA, Papademetriou V, Geladari CV, Kolyvas GN, Angelopoulos ET, Aronis KN. Home, automated office, and conventional office blood pressure as predictors of cardiovascular risk. J Am Soc Hypertens 2017;11:16570.e2.

18. Cordonnier C, Potter GM, Jackson CA, Doubal F, Keir S, Sudlow CL, Wardlaw JM, Al-Shahi Salman R. improving interrater agreement about brain microbleeds: development of the Brain Observer MicroBleed Scale (BOMBS). Stroke 2009;40:94-9.

19. Amin Al Olama A, Wason JMS, Tuladhar AM, van Leijsen EMC, Koini M, Hofer E, Morris RG, Schmidt R, de Leeuw FE, Markus HS. Simple MRI score aids prediction of dementia in cerebral small vessel disease. Neurology 2020;94:e1294-302.

20. Lee SH, Bae HJ, Yoon BW, Kim H, Kim DE, Roh JK. Low concentration of serum total cholesterol is associated with multifocal signal loss lesions on gradient-echo magnetic resonance imaging: analysis of risk factors for multifocal signal loss lesions. Stroke 2002;33:2845-9.

21. Wardlaw JM, Smith EE, Biessels GJ, Cordonnier C, Fazekas F, Frayne R, et al. Neuroimaging standards for research into small vessel disease and its contribution to ageing and neurodegeneration. Lancet Neurol 2013;12:822-38.

22. Fazekas F, Chawluk JB, Alavi A, Hurtig HI, Zimmerman RA. MR signal abnormalities at $1.5 \mathrm{~T}$ in Alzheimer's dementia and normal aging. AJR Am J Roentgenol 1987;149:351-6.

23. Haller S, Vernooij MW, Kuijer JPA, Larsson EM, Jäger HR, Barkhof F. Cerebral Microbleeds: Imaging and Clinical Significance. Radiology 2018;287:11-28.

24. Tanaka A, Ueno Y, Nakayama Y, Takano K, Takebayashi S. Small chronic hemorrhages and ischemic lesions in association with spontaneous intracerebral hematomas. Stroke 1999;30:1637-42.

25. Fan YH, Zhang L, Lam WW, Mok VC, Wong KS. Cerebral microbleeds as a risk factor for subsequent intracerebral hemorrhages among patients with acute ischemic stroke. Stroke 2003;34:2459-62.

26. Boulanger JM, Coutts SB, Eliasziw M, Gagnon AJ, Simon JE, Subramaniam S, Sohn CH, Scott J, Demchuk AM. Cerebral microhemorrhages predict new disabling or fatal strokes in patients with acute ischemic stroke or transient ischemic attack. Stroke 2006;37:911-4.

27. Nakaya M, Hashimoto H, Usui G, Sawada K, Shirouzu I, Oshima A, Okubo S, Yamada H, Morikawa T. Cerebral amyloid-beta-related angiitis without cerebral microbleeds in a patient with subarachnoid hemorrhage. Cardiovascular Pathology 2019;42:36-40.

28. Chen YF, Chang YY, Liu JS, Lui CC, Kao YF, Lan MY. Association between cerebral microbleeds and prior primary intracerebral hemorrhage in ischemic stroke patients. Clin Neurol Neurosurg 2008;110:988-91.

29. Henskens LH, van Oostenbrugge RJ, Kroon AA, de Leeuw PW, Lodder J. Brain microbleeds are associated with ambulatory blood pressure levels in a hypertensive population. Hypertension 2008;51:62-8.

30. Bokura H, Saika R, Yamaguchi T, Nagai A, Oguro H, Kobayashi S, Yamaguchi S. Microbleeds are associated with subsequent hemorrhagic and ischemic stroke in healthy elderly individuals. Stroke 2011;42:1867-71.

31. Nishikawa T, Ueba T, Kajiwara M, Fujisawa I, Miyamatsu N, Yamashita K. Cerebral microbleeds predict firstever symptomatic cerebrovascular events. Clin Neurol Neurosurg 2009;111:825-8.

32. Tsushima Y, Tanizaki Y, Aoki J, Endo K. MR detection of microhemorrhages in neurologically healthy adults. Neuroradiology 2002;44:31-6.

33. Viswanathan A, Chabriat H. Cerebral microhemorrhage. Stroke 2006;37:550-5.

34. Haacke EM, Xu Y, Cheng YC, Reichenbach JR. Susceptibility weighted imaging (SWI). Magn Reson Med 2004;52:612-8.

35. Sehgal V, Delproposto Z, Haacke EM, Tong KA, Wycliffe N, Kido DK, Xu Y, Neelavalli J, Haddar D, Reichenbach JR. Clinical applications of neuroimaging with susceptibility-weighted imaging. J Magn Reson Imaging 2005;22:439-50.

36. Jeerakathil T, Wolf PA, Beiser A, Massaro J, Seshadri S, D'Agostino RB, DeCarli C. Stroke risk profile predicts white matter hyperintensity volume: the Framingham Study. Stroke 2004;35:1857-61.

37. Naka H, Nomura E, Wakabayashi S, Kajikawa H, Kohriyama T, Mimori Y, Nakamura S, Matsumoto M. Frequency of asymptomatic microbleeds on $\mathrm{T} 2{ }^{*}$-weighted MR images of patients with recurrent stroke: association with combination of stroke subtypes and leukoaraiosis. AJNR Am J Neuroradiol 2004;25:714-9.

38. Gao T, Wang Y, Zhang Z. Silent cerebral microbleeds on susceptibility-weighted imaging of patients with ischemic 
stroke and leukoaraiosis. Neurol Res 2008;30:272-6.

39. Lee SH, Park JM, Kwon SJ, Kim H, Kim YH, Roh JK, Yoon BW. Left ventricular hypertrophy is associated with cerebral microbleeds in hypertensive patients. Neurology 2004;63:16-21.

40. Masuda J, Tanaka K, Ueda K, Omae T. Autopsy study of incidence and distribution of cerebral amyloid angiopathy in Hisayama, Japan. Stroke 1988;19:205-10.

41. Jensen-Kondering UR, Weiler C, Langguth P, Larsen N, Flüh C, Kuhlenbäumer G, Jansen O, Margraf NG. Clinical and radiological differences between patients with probable cerebral amyloid angiopathy and mixed cerebral microbleeds. J Neurol 2020;267:3602-8.

42. Tsushima Y, Aoki J, Endo K. Brain microhemorrhages detected on T2*-weighted gradient-echo MR images. AJNR Am J Neuroradiol 2003;24:88-96.

43. Shoamanesh A, Preis SR, Beiser AS, Vasan RS, Benjamin EJ, Kase CS, Wolf PA, DeCarli C, Romero JR, Seshadri S. Inflammatory biomarkers, cerebral microbleeds, and

Cite this article as: Liang C, Wang J, Feng M, Zhang N, Guo L. White matter changes, duration of hypertension, and age are associated with cerebral microbleeds in patients with different stages of hypertension. Quant Imaging Med Surg 2022;12(1):119-130. doi: 10.21037/qims-21-28 small vessel disease: Framingham Heart Study. Neurology 2015;84:825-32.

44. Seo SW, Hwa Lee B, Kim EJ, Chin J, Sun Cho Y, Yoon U, $\mathrm{Na}$ DL. Clinical significance of microbleeds in subcortical vascular dementia. Stroke 2007;38:1949-51.

45. Chiang GC, Cruz Hernandez JC, Kantarci K, Jack CR, Jr., Weiner MW, Alzheimer's Disease Neuroimaging I. Cerebral Microbleeds, CSF p-Tau, and Cognitive Decline: Significance of Anatomic Distribution. AJNR Am J Neuroradiol 2015;36:1635-41.

46. Li X, Yuan J, Yang L, Qin W, Yang S, Li Y, Fan H, Hu $W$. The significant effects of cerebral microbleeds on cognitive dysfunction: An updated meta-analysis. PLoS One 2017;12:e0185145.

47. Charidimou A, Imaizumi T, Moulin S, Biffi A, Samarasekera N, Yakushiji Y, et al. Brain hemorrhage recurrence, small vessel disease type, and cerebral microbleeds: A meta-analysis. Neurology 2017;89:820-9. 